\title{
Structural and Functional Studies on
}

\section{Hemoglobin Bethesda $\left(\alpha_{2} \beta_{2}{ }^{145 \text { His }}\right)$, a Variant Associated with Compensatory Erythrocytosis}

\author{
H. Franklin Bunn, Thomas B. Bradley, William E. Davis, \\ James W. Drysdale, John F. Burke, William S. Beck, and \\ MYRON B. LAVER \\ From the Thorndike Memorial Laboratory, Harvard Medical Service, Boston \\ City Hospital, Boston, Massachusetts 02118, Department of Medicine, \\ Veterans Administration Hospital, University of California, San Francisco, \\ California 94121, and Shriners Burns Institute and the Departments of \\ Medicine and Anesthesia, Massachusetts General Hospital, Harvard \\ Medical School, Boston, Massachusetts 02114
}

A в S T R A C T Studies have been performed on a 12 -yrold Chinese girl with compensatory erythrocytosis due to the presence of hemoglobin Bethesda comprising about $45 \%$ of the red cell hemoglobin. Her parents and three siblings were normal. The oxygen affinity of her blood was markedly increased: under physiological conditions $\left(\mathrm{pH} 7.40,37^{\circ} \mathrm{C}\right), \mathrm{P}_{50}$ was $12.8 \mathrm{~mm} \mathrm{Hg}$ (normal = 26.5 $\mathrm{mm} \mathrm{Hg}$ ). The red cell 2,3-diphosphoglycerate (2,3-DPG) level was normal. The abnormal hemoglobin could not be separated from hemoglobin A by zone electrophoresis at $\mathrm{pH} 8.6$ or isoelectric focusing on polyacrylamide gel. However, after the hemoglobin was split into free $\alpha$ and $\beta$ chains by treatment with $p$-hydroxymercuribenzoate (PMB) or $6 \mathrm{M}$ urea, an abnormal $\beta$ chain was readily demonstrated having a higher isoelectric point (more positive net charge) than normal $\boldsymbol{\beta}_{\boldsymbol{\Delta}}$. Structural analysis of the variant $\boldsymbol{\beta}$ chain demonstrated the substitution of histidine for tyrosine at position 145: hemoglobin Bethesda $\left(\alpha_{2} \beta_{2}{ }^{145 \mathrm{H} / \mathrm{s}}\right)$. From earlier chemical and crystallographic studies, it has been postulated that this residue is a critical determinant of hemoglobin function. Hemoglobin Bethesda was separated from hemoglobin A by column chromatography. Oxygen equilibria of purified hemoglobin Bethesda revealed an extremely high oxygen affinity (exceeding that of isolated $\alpha$ and $\beta$ chains), and

This paper was presented in part at the Annual Meeting of the American Society of Hematology, December 1971 (1).

Received for publication 3 March 1972 and in revised form 5 April 1972. markedly reduced cooperativity. The Bohr effect of hemoglobin Bethesda was $1 / 3$ that of hemoglobin A. However, hemoglobin Bethesda showed a significant interaction with 2,3-DPG and inositol hexaphosphate.

\section{INTRODUCTION}

Although most human hemoglobin variants appear to be functionally similar to hemoglobin A, an increasing number with markedly abnormal oxygen-binding properties have been found. Individuals heterozygous for a hemoglobin variant of unusually high oxygen affinity generally have compensatory erythrocytosis. This clinical phenomenon has provided new information on the physiological relationship between oxygen delivery and regulation of red cell mass.

In this report we present structural and functional information on a hemoglobin of high oxygen affinity, found in an asymptomatic Chinese girl with erythrocytosis. This hemoglobin variant has been found to be structurally identical with hemoglobin Bethesda $\left(\alpha_{2} \beta_{2}{ }^{145 \mathrm{Tyr}} \rightarrow{ }^{\mathrm{H} 1 \mathrm{~s}}\right)$ reported recently by Hayashi, Stamatoyannopoulos, Yoshida, and Adamson (2). Functional studies of this hemoglobin are of considerable relevance since recent X-ray crystallographic studies of Perutz have assigned considerable importance to this residue (3). Furthermore, our patient is of additional interest since her abnormal hemoglobin appears to have arisen because of a spontaneous mutation. A preliminary report of this work has appeared elsewhere (1). 


\section{METHODS}

Blood specimens were collected in either heparin or acid citrate dextrose (ACD).$^{1}$ Routine hematologic studies were done by standard methods. Red Cell 2,3-diphosphoglycerate (2,3-DPG) was determined by the method of Keitt (4) and Lowry, Passonneau, Hasselberger, and Schulz (5). Hemolysates were prepared according to Drabkin (6). Alkali-resistant hemoglobin was measured by the method of Singer, Chernoff, and Singer (7). Hemoglobin solutions were analyzed by zone electrophoresis on starch gel and cellulose acetate at $\mathrm{pH} 8.6$, and also by isoelectric focusing in polyacrylamide gel as described previously (8). Hemoglobins A and Bethesda were separated in the cold by chromatography on carboxymethylcellulose (CM-cellulose). ${ }^{2}$ The resin was equilibrated with $0.01 \mathrm{M}$ phosphate buffer, $\mathrm{pH}$ 7.0. After overnight dialysis of the hemolysate against this buffer, 600 $\mathrm{mg}$ of hemoglobin was applied to the column $(2.5 \times 25 \mathrm{~cm})$. A linear gradient was established by $800 \mathrm{ml}$ of the equilibrating buffer in the mixing vessel and an equal volume of $0.01 \mathrm{M}$ phosphate, $\mathrm{pH} 7.9$, in the inflow vessel. A flow rate of $20 \mathrm{ml} / \mathrm{hr}$ was maintained. Hemoglobin-rich peaks were concentrated by pressure dialysis ${ }^{3}$ and dialyzed vs. $0.1 \mathrm{M} \mathrm{NaCl}$, $0.01 \mathrm{~m}$ Tris buffer, $\mathrm{pH} 8.0$, before study. Isolated heme-intact $\alpha$ and $\beta$ chains were prepared from normal human hemolysate by the method of Bucci and Fronticelli (9). The globin thiols were regenerated as described by Geraci, Parkhurst, and Gibson (10).

Structural analysis. The methods outlined by Clegg, Naughton, and Weatherall (11) were followed with minor modifications for the preparation of globin, separation of the abnormal $\beta$ globin from the normal $\alpha$ and $\beta$ chains on CMcellulose columns in the presence of urea and mercaptoethanol, tryptic digestion, peptide mapping, elution of the peptides from the peptide maps, and acid hydrolysis. Aminoethylation of the $\beta$ globin before tryptic digestion was performed initially but proved to be unnecessary for these studies. The acid hydrolysates were dried by flash evaporation, dissolved in citrate buffer, $\mathrm{pH} 2.2$, and applied to a Beckman/Spinco 120C Amino Acid Analyzer (Beckman Instruments, Inc., Spinzo Div., Palo Alto, Calif.)

The amino acid sequence of the carboxyterminal portion of isolated $\beta$ globin chains was determined by digestion with carboxypeptidase A (CPA-DFP, Sigma Chemical Co., St. Louis, Mo., 20 units/mg protein) (12). A substrate to enzyme ratio of $150: 1$ was employed. The reaction was carried out in $0.025 \mathrm{M}$ barbital buffer, $\mathrm{pH} 8.8$, at $25^{\circ} \mathrm{C}$. Isoleucine was added as an internal standard before the addition of the enzyme and a sample removed for acid hydrolysis and amino acid analysis. Samples were removed from the reaction mixture at intervals and the reaction was stopped by the addition of 0.4 volumes of cold glacial acetic acid. These solutions were applied directly to the amino acid analyzer.

Oxygen equilibria. Oxygen equilibrium curves were determined on whole blood and concentrated hemoglobin solutions by the method of Duvelleroy, Buckles, Rosenkaimer, Tung, and Laver (13). A specimen of deoxygenated whole blood is exposed to a known quantity of oxygen in a closed chamber, and continuous simultaneous recording of blood and gas phase $\mathrm{Po}_{2}$ permit a display of blood $\mathrm{Po}_{2}$ vs. $\mathrm{O}_{2}$ content on an X-Y recorder from zero to full saturation. Spec-

${ }^{1}$ Abbreviations used in this paper: ACD, acid citrate dextrose; 2,3-DPG, 2,3-diphosphoglycerate; PMB, p-hydroxymercuribenzoate; $\beta \mathrm{Tp} \mathrm{XV}$, tryptic peptide XV.

${ }^{2} \mathrm{CM}-52, \mathrm{H}$. Reeve Angel and Co., Inc., Clifton, N. J.

${ }^{3}$ Amicon Corp., Lexington, Mass.

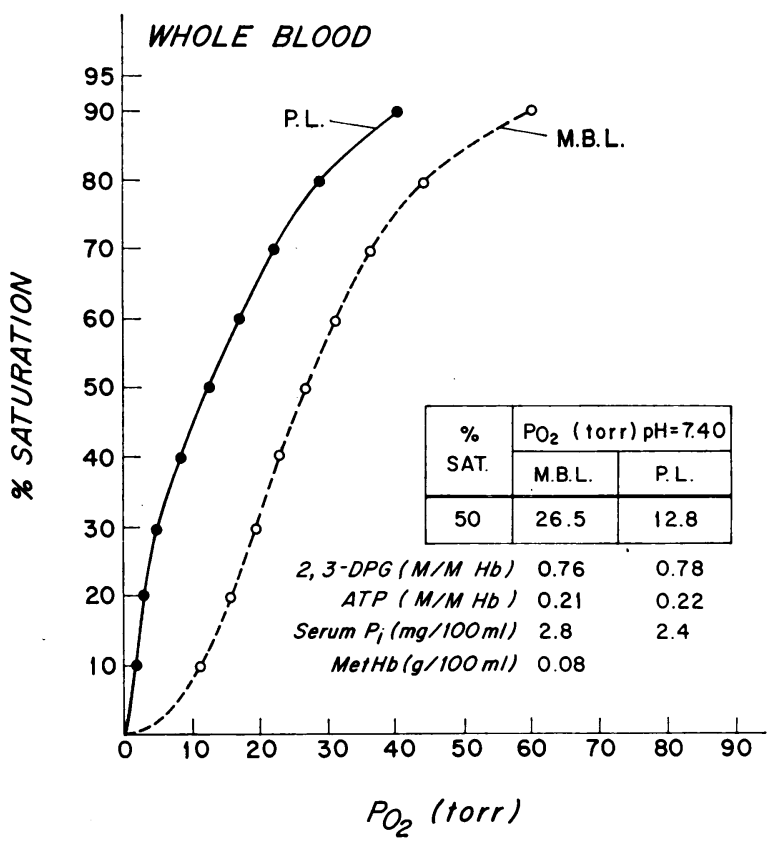

FIGURE 1 Oxygen saturation curves done on the whole blood of the proposita P. L. (- ) and a normal individual M. B. L. (O---O). In addition, values for red cell 2,3DPG, ATP, methemoglobin, and serum inorganic phosphates are shown.

trophotometric oxygen equilibria were determined on dilute hemoglobin solutions $(0.1 \mathrm{~mm}$ tetramer $)$ as described previously (14). Solutions of high oxygen affinity were deoxygenated by the passage of $99.997 \%$ nitrogen through a hydrator and then through the tonometer, which was shaking gently at room temperature. More precise oxygen saturation curves could be obtained on hemoglobins of high affinity if measured amounts of $3.20 \%$ oxygen ( $96.8 \%$ nitrogen) rather than room air were introduced into the tonometer. The oxygen content of this gas had been determined both by Scholander analysis and by its partial pressure of oxygen, measured with an appropriate $\mathrm{O}_{2}$ electrode. ${ }^{4}$ Conversely, in testing solutions of very low oxygen affinity, measured amounts of $100 \%$ oxygen were introduced into the tonometer. Standard solutions of 2,3-DPG were prepared as described previously (14). Inositol hexaphosphate was purchased from Sigma Chemical Co. It was used without further purification. Standard solutions were titrated to neutrality before use.

\section{RESULTS}

\section{Clinical and family data}

P. L., a 12-yr-old Chinese girl, was admitted to the Shriners Burns Institute because of second and third degree burns involving $10 \%$ of body surface. Physical examination was otherwise normal. During routine hematological examination she was found to have erythrocytosis. During the subsequent 5 months, hematocrit values ranged from 52 to 56 . (Normal hematocrit for a 12-yr-old girl is 39 [15].) The reticulocyte count was

\footnotetext{
"Instrumentation Laboratory, Inc., Lexington, Mass.
} 
TABLE I

Hematologic Data on P. L.'s Family

\begin{tabular}{lcccc}
\hline & Hematocrit & Hemoglobin & $\begin{array}{c}\text { PMB-treated } \\
\text { hemolysate* }\end{array}$ & $\begin{array}{c}\text { P of } \\
\text { phosphate-free } \\
\text { hemolysatef }\end{array}$ \\
\hline P. L. & & $\mathrm{g} / 100 \mathrm{ml}$ & & $\mathrm{mm} \mathrm{Hg}$ \\
Mother L. L. & 55 & 17.7 & Abnormal $\beta$ chain & 1.35 \\
Father R. L. & 50 & 14.9 & Normal & 3.85 \\
Sister M. L. & 44 & 16.7 & Normal & 3.41 \\
Brother K. L. & 39 & 14.5 & Not done & 3.90 \\
Brother D. L. & 37 & 12.9 & Not done & 3.85 \\
\hline
\end{tabular}

* Analyzed by isoelectric focusing on polyacrylamide gel (Fig. 2).

$\ddagger 0.1 \mathrm{mM}$ hemoglobin in $0.1 \mathrm{M} \mathrm{Cl}^{-}$, bis-Tris buffer, $\mathrm{pH} 7.2,20^{\circ} \mathrm{C}$.

2.2\%. The red cell indices and white cell and platelet counts were within normal limits. Morphology of peripheral blood cells was also normal. Hemoglobin electrophoresis on paper, starch gel, and cellulose acetate at $\mathrm{pH} 8.6$ showed no abnormal components. Alkali-resistant hemoglobin was $2 \%$ of the total. No unstable hemoglobin could be demonstrated by either Heinz body preparations or by incubating the patient's hemolysate at $55^{\circ} \mathrm{C}(16)$.

Because of the patient's unexplained erythrocytosis, oxygen equilibrium of the whole blood was determined and found to be markedly abnormal (Fig. 1). Red cell 2,3-DPG was $4.5 \mathrm{mmoles} /$ liter packed red cells (normal range: 4-5 $\mathrm{mm}$ ). Red cell ATP was also within normal limits. Oxygen equilibria done on hemolysate "stripped" of all organic phosphates (17) confirmed the presence of hemoglobin of high oxygen affinity (Table I). In contrast oxygen equilibria done on stripped hemolysates of both parents and all three siblings were within normal limits. Neither paternity nor maternity could be excluded by a comparison of 27 red cells and 7 serum antigens in P. L. and her parents. ${ }^{5}$

\section{Isolation of hemoglobin variant}

Since these results suggested that the red cells of the proposita contained a functionally abnormal hemoglobin, attempts were made to isolate it. Because no abnormal hemoglobin could be detected by zone electrophoresis at $\mathrm{pH} 8.6$, the hemolysate was analyzed by isoelectric focusing on polyacrylamide gel. This technique, which has very high resolution, has proven effective in separating other hemoglobins (8). As shown in Fig. 2, the pattern was indistinguishable from that of a normal hemolysate. However, after P. L.'s hemoglobin was split into $\alpha$ and $\beta$ subunits with $p$-hydroxymercuribenzoate

${ }^{5}$ Blood Grouping Laboratory, Boston, Mass. The following red cell antigens were tested: $\mathrm{A}_{1}, \mathrm{~A}, \mathrm{H}, \mathrm{B}, \mathrm{D}, \mathrm{C}, \mathrm{E}, \mathrm{c}, \mathrm{e}, \mathrm{C}^{\mathrm{w}}$, $\mathrm{K}, \mathrm{k}, \mathrm{Kp}^{\mathrm{a}}, \mathrm{Kp}^{\mathrm{b}}, \mathrm{Le}^{\mathrm{a}}$, Le $\mathrm{L}^{\mathrm{b}}, \mathrm{M}, \mathrm{N}, \mathrm{S}, \mathrm{s}, \mathrm{Lu}^{\mathrm{a}}, \mathrm{Lu}^{\mathrm{b}}, \mathrm{Fy}^{\mathbf{a}}$, $\mathrm{Jk}^{\mathbf{2}}, \mathrm{Wr}^{\mathrm{a}}, \mathrm{Vel}, \mathrm{Yt}^{\mathrm{a}}$. The following serum antigens were tested: $\mathrm{Hp}, \mathrm{Gm}^{*}, \mathrm{Gm}^{\mathrm{b}}, \mathrm{Gm}^{\mathrm{x}}, \mathrm{Gm}^{\mathrm{*}}, \mathrm{Gm}^{\mathrm{s}}$, $\mathrm{Inv}^{*}$.
(PMB) (9), isoelectric focusing (but not electrophoresis on cellulose acetate) revealed the presence of a second $\beta$ chain approximately equal in amount to the normal $\beta$ chain but having a higher isoelectric point (Fig. 2). The abnormal $\beta$ chain could not be detected in the PMB-treated hemolysate of either parent. P. L.'s abnormal $\beta$ chain could also be demonstrated and isolated from heme-free globin as described below. The variant tetramer could be separated from hemoglobin A by chromatography of P. L.'s hemolysate on CM-cellulose. A representative elution pattern is shown in Fig. 3.-These purified hemoglobins contained less than $5 \%$ methemoglobin.

\section{Structural studies}

When globin was separated on "urea columns," the abnormal $\beta$ globin was eluted just after the normal $\beta$ chains. The normal $\beta$ globin served as a control for the structural studies.

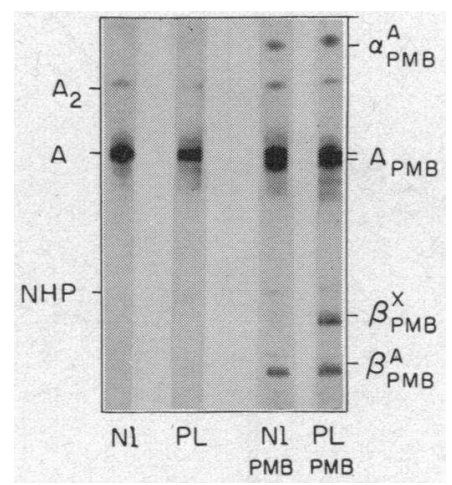

Figure 2 Separations of hemoglobins by isoelectric focusing on polyacrylamide gel. A linear $\mathrm{pH}$ gradient between 6 and 8 was used. Gels were stained with Coomassie blue. $\mathrm{Nl}$, normal hemolysate. PL, hemolysate of proposita. N1 $\mathrm{PMB}$ and $\mathrm{PL}$ PMB designate the same hemolysates after incubation with $p$-hydroxymercuribenzoate. NHP, nonheme protein. 


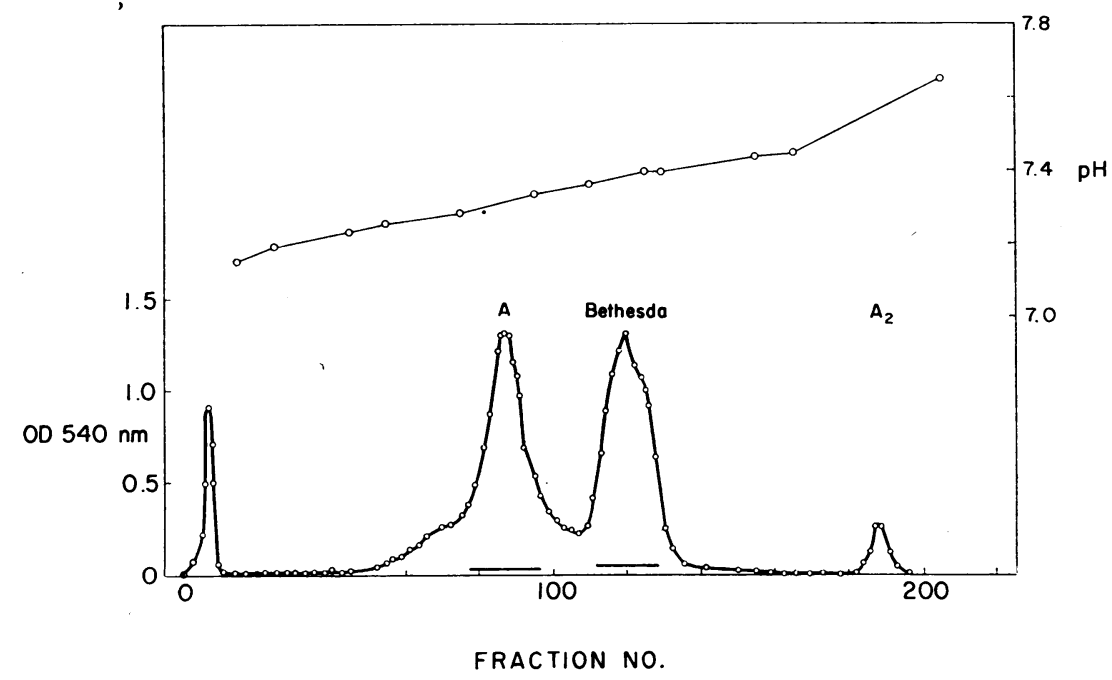

FigURE 3 Separation of hemoglobin A and hemoglobin Bethesda from hemolysate of proposita by column chromatography on CM-cellulose. The $\mathrm{pH}$ gradient is shown above.

Tryptic peptide XV ( $\beta$ Tp XV) was absent from the peptide maps of the abnormal $\beta$ chain. This dipeptide contains the carboxyterminal histidine and the penultimate residue tyrosine. A new, ninhydrin-reactive peptide was shown by staining techniques to contain histidine but not tyrosine. Amino acid analysis of the acid-hydrolysed abnormal peptide revealed only histidine. No residues
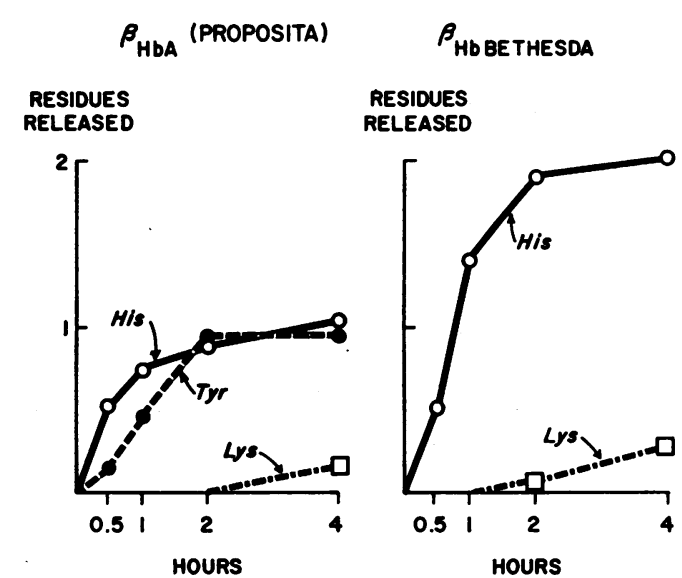

FIGURE 4 Carboxypeptidase A digestion of the normal and abnormal $\beta$ globin polypeptide chains of the proposita. The residues were released sequentially from the carboxyterminal end of the globin chain with the reaction slowing at the basic residue lysine $(\beta 144)$. The normal $\beta$ chain showed the expected sequence: histidine-tyrosine-lysine. With the abnormal $\beta$ chain, two residues of histidine were released before the lysine indicating that histidine had been substituted for the penultimate $(\beta 145)$ tyrosine. were resolved when the unhydrolysed peptide was analyzed indicating that the abnormality was not a deletion of the tyrosine. Determination of the amino acid sequence of the carboxyterminal portion of the abnormal $\beta$ chain (Fig. 4) indicated that histidine had been substituted for the tyrosine of position 145 (helical residue $\mathrm{H} 23$ ). This abnormality is identical with that of hemoglobin Bethesda (2).

\section{Functional studies}

Whole blood and hemolysate. Fig. 1 shows an arithmetic plot of the oxygen affinity of whole blood of the proposita and a normal coauthor studied under physiological conditions. ${ }^{\circ}$ The oxygen affinity of the patient's blood was markedly increased with half-saturation at $12.8 \mathrm{~mm} \mathrm{Hg}$ contrasted with the normal of $26.5 \mathrm{~mm} \mathrm{Hg}$. These data were plotted on a double logarithmic scale according to the Hill equation:

$$
\log \left(\frac{Y}{1-Y}\right)=n \log \mathrm{Po}_{2}-n \log \mathrm{P}_{50}
$$

where $Y$ is the fractional saturation of hemoglobin with

- Correction of $\mathrm{pH}$ determined for the whole blood equilibrium curve in vitro at $37^{\circ} \mathrm{C}$ was applied according to the Severinghaus Blood Gas Calculator (18). Since data are not available on the red cell $\mathrm{pH}$ or the Bohr factor $\left(\Delta \log \mathrm{Po}_{2}\right.$ ) $\Delta \mathrm{pH})$ for P. L.'s blood, the value for $\mathrm{P}_{\mathrm{so}}$ at $\mathrm{pH} 7.4$ and the curve drawn for her blood in Fig. 1 is tentative. The measured $\mathrm{pH}$ was 7.37 at the time the curves were drawn and the maximum error in the calculation of $\mathrm{P}_{50}$ at $\mathrm{pH} 7.4$ is less than $1 \mathrm{~mm} \mathrm{Hg}$. 
oxygen, and $\mathrm{P}_{\mathrm{so}}$ is the partial pressure of oxygen at which hemoglobin is half-saturated $(Y=0.5)$. The slope of this plot, $n$, is an index of cooperativity between hemoglobin subunits. As shown in Fig. 5, such a plot of the oxygen saturation curve of normal blood is linear over a wide range of $Y$, with a $P_{50}$ of $26 \mathrm{~mm} \mathrm{Hg}$ and an $n$ of about 2.8. The Hill plot for P. L.'s blood shows a biphasic curve with an inflection point at about half-saturation (Fig. 5). This represents additional evidence for the presence within P. L.'s red cells of two hemoglobins: an abnormal hemoglobin with very high oxygen affinity and low cooperativity and an approximately equal amount of normal hemoglobin having normal oxygen affinity and full cooperativity. The unbroken line in Fig. 5 has been

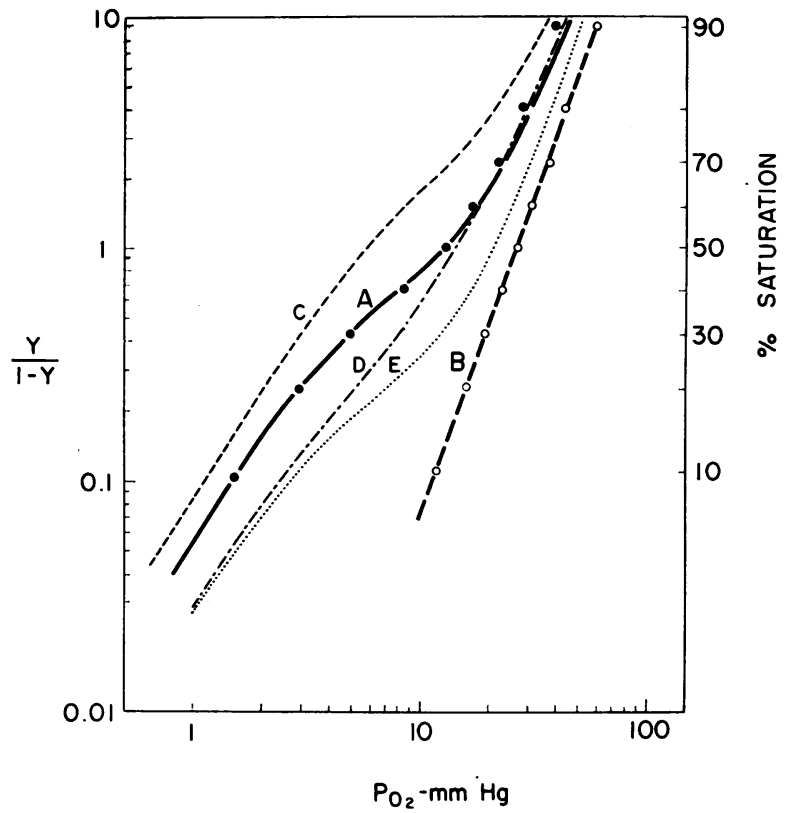

Figure 5 Hill plot of the data shown in Fig. 1. Proposita P. L. (๑), normal M. B. L. (O). The solid line A ( $\longrightarrow)$ represents a theoretical plot based on the assumption that two hemoglobins are present in equal amounts: hemoglobin A $\left(P_{s_{0}}=26.5 \mathrm{~mm} \mathrm{Hg}, n=2.8\right)$ and hemoglobin Bethesda $\left(P_{s 0}=3.8 \mathrm{~mm} \mathrm{Hg}, n=1.6\right)$. These values for hemoglobin $A$ are taken from the Hill plot of normal control M.B.L., curve $\mathrm{B}(\mathrm{O}---\mathrm{O})$. The $\mathrm{P}_{50}$ value for hemoglobin Bethesda was taken from the experimental $\mathrm{Po}_{2}$ at which $Y=0.25$. The $n$ value for hemoglobin Bethesda was taken from oxygen equilibria done on the purified hemoglobin in the presence of 2,3-DPG (see Table III). The plot ( $\longrightarrow$ ) is constructed on the assumption that the two hemoglobins are noninteracting; i.e., the hybrid $\alpha_{2} \beta^{\Delta} \beta^{\text {Bethesda }}$ is not favored. In contrast, if the hybrid were formed according to the binomial distribution $\left(\alpha_{2} \beta_{2}{ }^{\mathbf{A}}, 2 \alpha_{2} \beta^{\mathbf{A}} \beta^{\text {Bothesda }}, \alpha_{2} \beta_{2}^{\text {Botheoda }}\right.$ ) the following plots would be obtained: hybrid's functional properties are identical with those of hemoglobin Bethesda (curve $\mathrm{C},---$ ); of hemoglobin A, (curve E, $\cdots$ ); hybrid has oxygen affinity and cooperativity midway between that of the two parent hemoglobins (curve $\mathrm{D},-\cdots \cdot-\cdot-$ ).

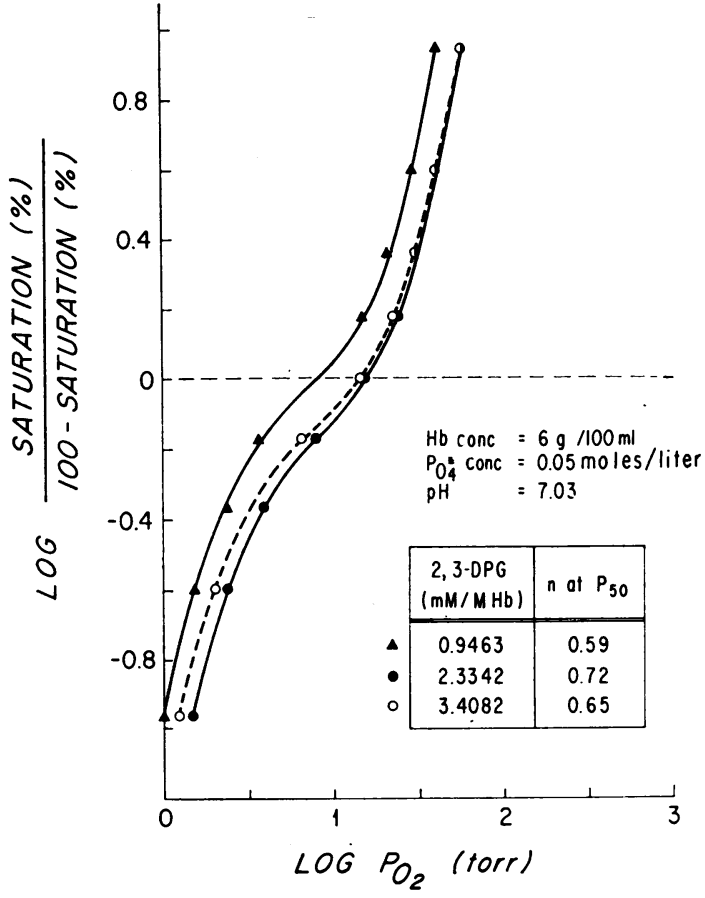

FIgURE 6 Hill plots of hemolysate of proposita P. L. to which 2,3-DPG has been added. Hemoglobin concentration, $6 \mathrm{~g} / 100 \mathrm{ml}$.

constructed on the assumption that two noninteracting hemoglobins are present in approximately equal amounts. It is a summation of two independent Hill equations: hemoglobin A ( $\left.\mathrm{P}_{5_{0}}=28 \mathrm{~mm} \mathrm{Hg}, n=2.8\right)$ and hemoglobin Bethesda ( $\left.P_{5_{0}}=3.7, n=1.6\right)$. The fact that the experimental data (curve A, solid dots) fit so well with this theoretical plot (curve A, solid line) indicates that the hybrid $\alpha_{2} \beta^{\boldsymbol{\Lambda}} \beta^{\text {Bethesda }}$ is not favored. If a significant amount of the hybrid were present, a different Hill plot would be expected (Fig. 5 ).

The effect of the addition of 2,3-DPG to a fresh hemolysate of P.L.'s blood is shown in Fig. 6. This experiment was done with $6 \mathrm{~g} / 100 \mathrm{ml}$ hemoglobin solutions in 0.05 m phosphate, $\mathrm{pH} 7.03,37^{\circ} \mathrm{C}$. It is apparent that 2,3-DPG effects more of a "rightward shift" on the upper portion of the curve than the lower portion. This indicates that the variant hemoglobin interacts with 2,3-DPG less strongly than hemoglobin A, a finding confirmed by studies on the isolated hemoglobins (see below).

Isolated hemoglobins. Visible spectra $(500-750 \mathrm{~nm})$ of the oxy- and deoxy-forms of hemoglobin Bethesda were normal. ${ }^{7}$ The absorbance ratio $540 \mathrm{~nm} / 280 \mathrm{~nm}$ was

\footnotetext{
'In contrast, the spectrum of deoxyhemoglobin Bethesda in the Soret region $(400-450 \mathrm{~nm})$ has been shown to be similar to that of isolated $\alpha$ and $\beta$ chains, rather than normal hemoglobin tetramer. (J. S. Olson, and Q. H. Gibson. 1972. J. Biol. Chem. $247:$ 3662.)
} 
TABLE II

Comparison of Oxygen Equilibria of Functionally Abnormal Hemoglobin Variants, Normal $(A)$ Hemoglobin and Isolated $\alpha$ and $\beta$ Chains*

\begin{tabular}{lcc}
\hline Hemoglobin & $\mathrm{P}_{\mathrm{s0}}$ & $n$ (at $Y=0.5$ ) \\
\hline & $m m \mathrm{Hg}$ & \\
Bethesda & 0.18 & 1.1 \\
$\beta^{\mathrm{SH}}$ & 0.24 & 1.0 \\
$\alpha^{\mathrm{SH}}$ & 0.45 & 1.0 \\
Chesapeake & $0.55 \ddagger$ & 1.4 \\
Hiroshima & 0.90 & 2.2 \\
A & 3.9 & 3.0 \\
\hline
\end{tabular}

* Arranged in order of decreasing oxygen affinity. $0.1 \mathrm{mM}$ (phosphate-free) hemoglobin in $0.1 \mathrm{M} \mathrm{Cl}^{-}$, bis-Tris buffer, $\mathrm{pH} 7.2$, $20^{\circ} \mathrm{C}$.

$\ddagger$ Oxygen equilibrium was done at $10^{\circ} \mathrm{C}$ (14). $\mathrm{P}_{50}$ of 0.28 was corrected to 0.55 at $20^{\circ} \mathrm{C}$ by applying correction factor determined for phosphate-free hemoglobin A (34).

identical for the variant and normal hemoglobins, indicating that hemoglobin Bethesda has four heme groups per tetramer (19).

Oxygen equilibria of hemoglobin A (and also hemoglobin $\mathrm{A}_{2}$ ) prepared from $P$. L.'s hemolysate showed the expected low oxygen affinity and normal heme-heme interaction $(n=2.8)$. In contrast, the oxygen affinity of hemoglobin Bethesda was very high, exceeding that of isolated $\alpha$ or $\beta$ chains (Table II and Fig. 7). Further- more, like isolated $\alpha$ (and $\beta$ ) chains, hemoglobin Bethesda lacked cooperativity. The ends of the saturation curve $(Y<0.4$ and $Y>0.6)$ approached an $n$ of 1.0 . The pattern of these Hill plots was reproducible on repeat experiments. The proximity of these asymptotes to one another indicates a very low energy of interaction between subunits (20). At the extremes of oxygen saturation $(Y<0.05$ and $Y>0.95)$ hemoglobin A also approaches an $n$ of 1.0 (20). The much greater distance between these asymptotes reflects a correspondingly greater degree of subunit interaction. The oxygen affinity of hemoglobin Bethesda was little affected by $\mathrm{pH}$ (Fig. 8). The alkaline Bohr effect, expressed quantitatively as $\Delta \log \mathrm{P}_{\mathrm{s}_{0}} / \Delta \mathrm{pH}$, was $1 / 3$ normal: -0.21 vs. -0.57 . As shown in Table III, the oxygen affinity of hemoglobin Bethesda was appreciably lowered by 2,3DPG and inositol hexaphosphate although to a lesser extent than hemoglobin A. Furthermore, the presence of these organic phosphates brought about a significant increase in subunit cooperativity as estimated by Hill's $n$.

\section{DISCUSSION}

The usual criteria indicating the presence of a hemoglobin variant are the presence of an abnormal band on zone electrophoresis in conjunction with positive family studies. These were both lacking in our patient. The abnormal whole blood oxygen saturation curve provided the first evidence that P. L.'s erythrocytosis was due to the presence of a functionally abnormal hemoglobin. The

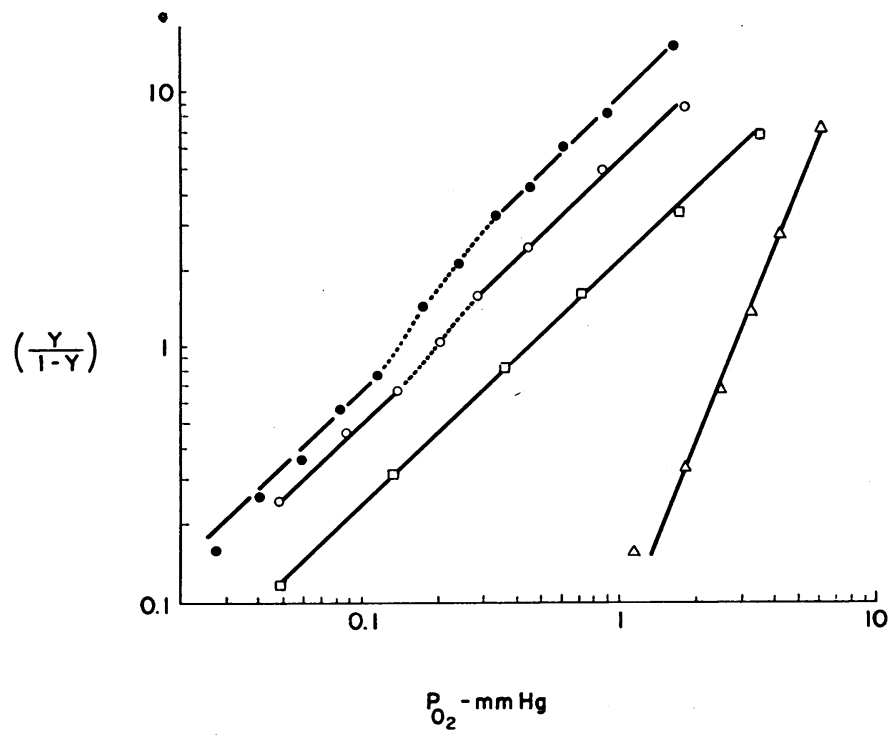

FIGURE 7 Hill plots of hemoglobins isolated by chromatography on CM-cellulose. Oxygen equilibria were done on $0.1 \mathrm{~mm}$ hemoglobin solutions in $0.1 \mathrm{M} \mathrm{Cl}^{-}$, and $0.05 \mathrm{M}$ bis-Tris- $\mathrm{HCl}$ or Tris- $\mathrm{HCl}$ buffer, $20^{\circ} \mathrm{C}$. Hemoglobin Bethesda, pH 7.6 (•-๑); hemoglobin Bethesda, pH 6.4 ( $\bigcirc-\mathrm{O})$; $\alpha$ chains, $\mathrm{pH} 7.2$ ( $\square-\square)$; hemoglobin A, pH $7.2(\triangle-\triangle)$. 


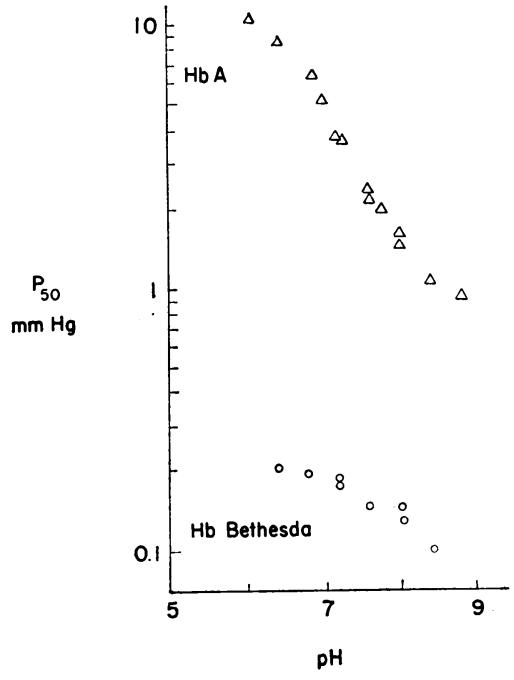

Figure 8 Bohr effect of hemoglobins $A(\triangle)$ and Bethesda (O). Experimental conditions as stated in the legend of Fig. 7. $P_{50}$ values are plotted on a logarithmic scale against $\mathrm{pH}$.

oxygen affinity, as depicted by the marked left shift of the oxygen saturation curve (Fig. 1), is as high as any reported to date for human blood $(21,22)$. Initial attempts to demonstrate a hemoglobin variant in P. L.'s hemolysate were unsuccessful. ${ }^{8}$ Only when her hemoglobin was split into $\alpha$ and $\beta$ chain subunits could a mutant $\beta$ chain be demonstrated. Fortunately, the variant hemoglobin in its native (tetrameric) form could be isolated by column chromatography. As with most other $\beta$ chain variants, it comprised about half of the hemoglobin in the red cell hemolysate.

Genetic considerations. Studies of the red cell and serum antigens of the proposita and her parents strongly suggest that the abnormal $\beta$ chain arose in the proposita as the result of a spontaneous mutation in a parental germ cell or early in embryogenesis. 20 other examples of possible spontaneous mutations in human hemoglobin have been reported (Table IV). With the exception of $\mathrm{Hb}$ Freiburg, where a valine was deleted from the $\beta$ chains, these mutations represented single amino acid substitutions and can be accounted for in each case by a change in a single base of a triplet codon.

\footnotetext{
${ }^{8}$ Hayashi and his associates were able to separate hemoglobins $\mathrm{A}$ and Bethesda by electrophoresis on agar gel, $\mathrm{pH}$ 6.0 (2).

An exception may exist for two hemoglobin variants with amino acid substitutions for valine $\beta 67$ (E11), hemoglobin Bristol ( $\beta 67 \mathrm{Asp})$, and $\mathrm{Hb} \mathrm{M}$-Milwaukee ( $\beta 67 \mathrm{Glu})$. There are four codons for valine, GUU, GUC, GUA, GUG. The codons for glutamic acid are GAA, GAG, and for aspartic acid are GAU, GAC. A single-step mutation to glutamic acid indicates that the codon for $\beta 67$ valine is either GUA or GUG. This precludes a single-step mutation from valine to aspartic acid at this position.
}

TABLE III

Effect of Organic Phosphates on the Oxygen Equilibria of Hemoglobins $A$ and Bethesda*

\begin{tabular}{|c|c|c|c|c|c|}
\hline \multirow[b]{2}{*}{ Organic phosphate } & \multirow[b]{2}{*}{$\mathrm{pH}$} & \multicolumn{2}{|c|}{$\mathbf{A}$} & \multicolumn{2}{|c|}{ Bethesda } \\
\hline & & $P_{\text {so }}$ & $n$ & $P_{\text {so }}$ & $n$ \\
\hline & & $n m H_{g}$ & & $m m H$ & \\
\hline None & 6.6 & 8.5 & 3.0 & 0.21 & 1.1 \\
\hline None & 7.2 & 3.9 & 3.1 & 0.18 & 1.2 \\
\hline $1 \mathrm{~mm} 2,3-\mathrm{DPG}$ & 6.6 & 22 & 2.6 & 0.52 & 1.4 \\
\hline $1 \mathrm{~mm} 2,3-\mathrm{DPG}$ & 7.2 & 9.4 & 2.9 & 0.29 & 1.6 \\
\hline $1 \mathrm{~mm}$ inositol hexaphosphate & 6.6 & 83 & 1.6 & 1.25 & 2.3 \\
\hline
\end{tabular}

* Experimental conditions as stated in legend to Fig. 7.

We have analyzed these substitutions in terms of the RNA genetic code. There were 14 pyrimidine transitions: 10 uracil to cytosine $(\mathrm{U} \rightarrow \mathrm{C}$ ) and 4 cytosine to uracil $(\mathrm{C} \rightarrow \mathrm{U})$. There were three purine transitions: two guanine to adenine $(\mathrm{G} \rightarrow \mathrm{A})$ and one adenine to guanine $(A \rightarrow G)$. Finally, there were three transversions (purine to pyrimidine or vice versa) $: G \rightarrow U, U \rightarrow G$, and $\mathrm{U} \rightarrow \mathrm{A}$. While both the small number of cases and the uncertainties regarding ancestry do not permit a firm analysis of these observations, it is noteworthy that the proportion of pyrimidine transitions in this series greatly exceeds that expected either from considerations of the genetic code or from the reported experience with human hemoglobin variants.

The classes of base replacements that account for the amino acid substitutions in 115 variants of hemoglobin $A$ are listed in Table $\mathrm{V}$. The proportions of the transitions and transversions that would be expected in the $\alpha$ and $\beta$ chains if base replacement were random cannot be calculated with precision because the specific codon for each amino acid is not known, but would approximate $15 \%$ for each of the two classes of transitions and $70 \%$ for the transversions. ${ }^{10}$ It is apparent from the data in Table $\mathrm{V}$ that there are substantial deviations from these expectations among the reported hemoglobin variants. A number of factors contribute to the observed proportion of the transitions and transversions.

The asymptomatic hemoglobin variants have largely been discovered by electrophoretic techniques during the course of population surveys. With three possible excep-

${ }^{10}$ The proportion of amino acid substitutions which would result from pyrimidine and purine transitions and transversions if mutation were random cannot be calculated with precision because the specific codon, of the several possible codons, is not known for each amino acid residue. We have calculated the range of mutations expected for each class of mutations in hemoglobin based on the amino acid composition of the $\alpha$ and $\beta$ globin chains and assuming that the $\alpha$ chain genetic locus has been duplicated (23). There are 428 codons at risk with 3852 possible one-step mutations. Of those which would result in amino acid substitutions, purine transitions would account for 418-479, pyrimidine transitions for 319-434, and transversions for 1885-2142. 
TABLE IV

Apparent Spontaneous Mutations among Hemoglobin Variants

\begin{tabular}{|c|c|c|c|c|c|}
\hline Hemoglobin & Structure & Clinical status & Blood antigens tested & $\begin{array}{l}\text { RNA base } \\
\text { change }\end{array}$ & Reference \\
\hline Hammersmith* & $\alpha_{2} \beta_{2}{ }^{42} \mathrm{Phe} \rightarrow \mathrm{Ser}$ & Hemolysis & No & $\mathrm{U} \rightarrow \mathrm{C}$ & 35 \\
\hline Köln $\ddagger$ & $\alpha_{2} \beta_{2}{ }^{98} \mathrm{Val} \rightarrow$ Meth & Hemolysis & Yes (36) & $\mathrm{G} \rightarrow \mathrm{A}$ & 37,36 \\
\hline Santa Ana & $\alpha_{2} \beta_{2}^{88 \mathrm{Leu} \rightarrow \text { Pro }}$ & Hemolysis & No & $\mathrm{U} \rightarrow \mathrm{C}$ & 37,38 \\
\hline Olmstead & $\alpha_{2} \beta_{2}{ }^{141}$ Leu $\rightarrow$ Arg & Hemolysis & No & $\mathrm{U} \rightarrow \mathrm{G}$ & 37 \\
\hline Freiburg & $\alpha_{2} \beta_{2}{ }^{23}$ Vel $\rightarrow 0$ & Hemolysis & No & & 39 \\
\hline Sabine & $\alpha_{2} \beta_{2}{ }^{11}$ Leu $\rightarrow$ Pro & Hemolysis & Yes & $\mathrm{U} \rightarrow \mathrm{C}$ & 40 \\
\hline Savannah & $\alpha_{2} \beta_{2}^{24 \mathrm{Gly} \rightarrow \mathrm{Val}}$ & Hemolysis & No & $\mathrm{G} \rightarrow \mathrm{U}$ & 41 \\
\hline Toulouse & $\alpha_{2} \beta_{2}{ }^{66 \mathrm{Lys} \rightarrow \mathrm{Glu}}$ & Hemolysis & No & $A \rightarrow G$ & 42 \\
\hline Bristol & $\alpha_{2} \beta_{2}{ }^{67}$ Val $\rightarrow$ Asp & Hemolysis & No & $\mathrm{U} \rightarrow \mathrm{A}$ & 43 \\
\hline Bryn Mawr & $\alpha_{2} \beta_{2}{ }^{85}$ Phe $\rightarrow$ Ser & Hemolysis & No & $\mathrm{U} \rightarrow \mathrm{C}$ & $\S$ \\
\hline Casper $\ddagger$ & $\alpha_{2} \beta_{2}{ }_{2}^{106}$ Leu $\rightarrow$ Pro & Hemolysis & Yes & $\mathrm{U} \rightarrow \mathrm{C}$ & 46 \\
\hline M-Saskatoon $\ddagger$ & $\alpha_{2} \beta_{2}{ }^{63} \mathrm{His} \rightarrow \mathrm{Tyr}$ & Cyanosis & Yes & $\mathrm{C} \rightarrow \mathrm{U}$ & 44,45 \\
\hline M-Hyde Park & $\alpha_{2} \beta_{2}{ }^{92} \mathrm{His} \rightarrow \mathrm{Tyr}$ & Cyanosis & Yes & $\mathrm{C} \rightarrow \mathrm{U}$ & 47 \\
\hline M-Boston & $\alpha_{2}{ }^{58 \mathrm{His} \rightarrow \mathrm{Tyr}} \beta_{2}$ & Cyanosis & No & $\mathrm{C} \rightarrow \mathrm{U}$ & 45 \\
\hline Bethesda & $\alpha_{2} \beta_{2}{ }^{145 \mathrm{Tyr} \rightarrow \mathrm{His}}$ & Erythrocytosis & Yes & $\mathrm{U} \rightarrow \mathrm{C}$ & This report \\
\hline
\end{tabular}

* Three instances.

$\ddagger$ Two instances.

§ Bradley, T. B., H. F. Bunn, and F. A. Oski. Unpublished data.

tions, amino acid substitutions due to pyrimidine transitions have been identified only when clinical manifestations have directed attention to the hemoglobins. The relatively high number of pyrimidine transitions among the unstable hemoglobin variants can possibly be explained by the nature of the amino acid substitutions which produce instability of the hemoglobin molecule (24). Conversely, many possible transversions and purine transitions may be lethal, and consequently undetectable, because they would lead to the introduction of a charged amino acid residue into the hydrophobic interior of the hemoglobin molecule. In general, only hydrophobic substitutions can be tolerated in this region of the molecule although they may produce instability of the hemoglobin by their failure to participate in normal bonding forces exerted over short distances with other amino acids and with the heme group. It is apparent from these brief considerations that no firm conclusions about the relative pathogenicity of the several classes of mutations can be derived from the distribution of these mutations among the symptomatic hemoglobin variants.

The large number of purine transitions has been shown by Fitch (25) and Vogel (26) to represent chiefly the replacement of guanine by adenine (RNA code). 25 of the 114 mutations summarized in Table $\mathrm{V}$ were of this type. The reverse mutation, adenine to guanine, occurred

TABLE V

Classes of Mutations among Variants of Hemoglobin A due to Single Amino Acid Substitutions

\begin{tabular}{lcccccr}
\hline & \multicolumn{2}{c}{ Transitions } & & & \\
& \multicolumn{1}{c}{ Pyrimidine } & Purine & & Transversions & $\begin{array}{c}\text { Purine transition } \\
\text { or transversion }\end{array}$ & Total \\
\hline$\alpha$ variants & 5 & 11 & 23 & 2 & 41 \\
$\beta$ variants & 12 & 28 & 31 & 3 & 74 \\
Total $\alpha$ and $\beta$ variants & 17 & 39 & 54 & 5 & 115 \\
Symptomatic variants* & 14 & 8 & 18 & 1 & 41 \\
$\quad(a)$ Unstable Hbs & 9 & 5 & 11 & 1 & 26 \\
(b) Hbs M & 4 & 0 & 1 & 0 & 5 \\
(c) Hbs, abnormal function & 1 & 3 & 6 & 0 & 10 \\
Asymptomatic variants & 3 & 31 & 36 & 4 & 74 \\
\hline
\end{tabular}

* The common variants which are chiefly symptomatic in the homozygous state or in combination with other hemoglobin abnormalities are excluded from this category; e.g. Hbs, S, C, D, E, and C-Harlem. 
in only 14 cases. These authors suggested that a higher probability existed for this particular mutational event, i.e., G $\rightarrow$ A. However, Ohta and Kimura (27) recently presented evidence that base mutation in hemoglobin is random and attributed the high proportion of guanine to adenine transitions to a differential survival of randomly occurring mutations. The proportion of the various types of mutations is influenced by the fitness of the hemoglobin variants and the limitations of the techniques employed to detect these variants. The relative frequency of the various types of mutations cannot be derived from such data.

It is not surprising that all of the reported "new" mutant hemoglobins had clinical manifestations since the symptomatic hemoglobins are drawn from a vastly larger population than the benign variants which are discovered during the course of population surveys. It is also probable that family studies are done more commonly when the variant is associated with disease. It cannot be concluded that spontaneous mutations are likely to be detrimental, although it is recognized that the decreased fitness of defective hemoglobins would cause the proportion of these variants to be higher among those discovered in the first generation than among those which have accumulated in the population. The fitness of the reported new mutations cannot be adequately assessed. Several, such as the $\mathrm{M}$ hemoglobins and hemoglobin Bethesda, would seem to pose little threat to the life expectancy of the affected individual although the impaired transfer of oxygen across the placenta may influence the reproductive potential of affected females.

Structural-functional relationships. Purified phosphate-free hemoglobin Bethesda had extraordinarily high oxygen affinity, greater than that of isolated $\alpha$ and $\beta$ chains, or any other hemoglobin variant thus far studied by ourselves (Table II) or others. Furthermore, this hemoglobin had very low cooperativity between subunits and decreased Bohr effect. It was possible that a significant amount of denaturation occurred during the column preparation. This seems unlikely, however, since hemoglobin $A$ recovered from the same column, had normal cooperativity $(n=2.8)$, and its oxygen affinity was only slightly higher than that of normal phosphatefree hemolysate. Also, a marked reduction in oxygen affinity and a significant increase in cooperativity was observed when hemoglobin Bethesda was studied in the presence of inositol hexaphosphate (see below). This cofactor lowers the oxygen affinity of hemoglobin even more effectively than 2,3-DPG (28).

It is useful to consider the abnormal functional behavior of hemoglobin Bethesda in terms of its structural mutation: $\beta^{145 \mathrm{Tgr}} \rightarrow^{\mathrm{H} 1 \mathrm{~s}}$. Recent high resolution X-ray crystallography data of Perutz (3) indicate that deoxyhemoglobin $\mathrm{A}$ is stabilized by specific constraints that are not energetically favorable when the molecule binds ligands such as oxygen. Among these are inter- and intrasubunit salt bonds, including specific residues responsible for the binding of 2,3-DPG and Bohr protons. The salt bonds are broken upon oxygenation. Furthermore, Perutz has presented evidence that in deoxyhemoglobin, the penultimate tyrosines of both $\alpha$ and $\beta$ chains are stabilized in pockets between the $F$ and $H$ helices in part by van der Waal's forces and in part by hydrogen bonding between the tyrosines' hydroxyls and the carbonyls of valines FG5. Upon oxygenation, these tyrosines are ejected and the $C$ terminal residues, now free of constraints, are able to rotate freely. In deoxyhemoglobin Bethesda the partially ionized histidine would be probably unable to occupy the hydrophobic pocket. This appears to prevent the formation of the oxygenlinked salt bridges of the $\beta$ carboxyterminal histidine. In normal hemoglobin $\mathrm{A}$, the bonding of its imidazole with the carboxyl group of $\beta 94$ aspartic acid is thought to account for about half of the alkaline Bohr effect (3). Thus, in deoxyhemoglobin Bethesda, the substitution of histidine for the penultimate tyrosine of the $\beta$ chains probably prevents adequate stabilization of the deoxy tetramer. In comparison to hemoglobin $\mathrm{A}$, the equilibrium between the constrained or deoxy- conformation and the relaxed or liganded conformation would be shifted in favor of the latter. These considerations probably account for the molecule's high oxygen affinity, low cooperativity, and decreased Bohr effect. Other hemoglobin variants with abnormally high oxygen affinity have also been viewed in terms of the equilibrium between deoxyand liganded conformations $(29,30)$. Further experimental data on hemoglobin Bethesda strengthens such an interpretation. Olson and Gibson have found that deoxyhemoglobin Bethesda dissociated reversibly into dimers $\left(\alpha_{2} \beta_{3} \rightleftharpoons 2 \alpha \beta\right)$ under conditions wherein deoxyhemoglobin A remained entirely tetrameric. However, in the presence of inositol hexaphosphate, the subunit dissociation of deoxyhemoglobin Bethesda was abolished. Furthermore, in the presence of this potent anionic cofactor, the molecule acquired some degree of functional integrity: the absorbence spectrum in the Soret (400-450 $\mathrm{nm}$ ) now resembled that of deoxyhemoglobin $A$ and there was kinetic evidence for cooperativity between subunits (see footnote 7 ).

It is interesting to compare hemoglobin Bethesda with hemoglobin Chesapeake, the first variant found in association with compensatory erythrocytosis $(31,32)$. It is likely that both hemoglobins have high ligand affinity because of a shift in the conformational equilibrium in favor of the liganded form. As postulated above, the amino acid substitution in hemoglobin Bethesda lessens the stability of the deoxy- form. In contrast, hemoglobin Chesapeake's substitution at the $\alpha_{1} \beta_{2}$ interface appears to result in increased stability of the liganded form $(33,30)$. 
Note added in proof: Recently, functional studies of hemoglobin Bethesda have been published. Hayashi, A., and G. Stamatoyannopoulos. 1972. Role of penultimate tyrosine in haemoglobin $\beta$ subunit. Nat. New Biol. 235: 71.

\section{ACKNOWLEDGMENTS}

The capable technical assistance of Charlotte Tung, Allison Chao, and Gail Kaufman is greatly appreciated.

Dr. Helen Ranney and Dr. Herman Lehmann provided some of the information used in Table IV.

This work was supported in part by Harvard Anaesthesia Center Grant GM No. 705-9711, by National Institutes of Health Grants HLO7497, AMO5391, HEO7652, and RR76, by Contract DADA17-70-C-0043 from the United States Army Medical Research and Development Command, and by the Veterans Administration.

\section{REFERENCES}

1. Bradley, T. B., H. F. Bunn, J. W. Drysdale, W. S. Beck, and M. B. Laver. 1971. Functional properties of hemoglobin Bethesda $\left(\alpha_{2} \beta_{2}^{145}{ }^{14 s}\right)$. Blood J. Hematol. 38: 794. (Abstr.)

2. Hayashi, A., G. Stamatoyannopoulos, A. Yoshida, and J. Adamson. 1971. Haemoglobin Rainier: $\beta 145$ (HC2) tyrosine $\rightarrow$ cysteine and haemoglobin Bethesda: $\beta 145$ (HC2) tyrosine $\rightarrow$ histidine. Nat. New Biol. 230: 264.

3. Perutz, M. F. 1970. Stereochemistry of cooperative effects in haemoglobin. Nature (Lond.). 228: 726.

4. Keitt, A. S. 1971. Reduced nicotinamide adenine dinucleotide-linked analysis of 2,3-diphosphoglyceric acid: spectrophotometric and fluorometric procedures. J. Lab. Clin. Med. $77: 470$.

5. Lowry, O. H., J. V. Passonneau, F. X. Hasselberger, and D. W. Schulz. 1964. Effect of ischemia on known substrates and cofactors of the glycolytic pathway in brain. J. Biol. Chem. 239: 18.

6. Drabkin, D. L. 1946. Spectrophotometric studies. XIV. The crystallographic and optical properties of the hemoglobin of man in comparison with those of other species. J. Biol. Chem. $164: 703$.

7. Singer, K., A. I. Chernoff, and L. Singer. 1951. Studies on abnormal hemoglobins. I. Their demonstration in sickle cell anemia and other hematologic disorders by means of alkali denaturation. Blood J. Hematol. 6: 413.

8. Drysdale, J. W., P. Righetti, and H. F. Bunn. 1971. The separation of human and animal hemoglobins by isoelectric focusing in polyacrylamide gel. Biochim. Biophys. Acta. 229: 42.

9. Bucci, E., and C. Fronticelli. 1964. A new method for the preparation of $\alpha$ and $\beta$ subunits of human hemoglobin. J. Biol. Chem. 240: PC 551.

10. Geraci, G., L. J. Parkhurst, and Q. H. Gibson. 1969. Preparation and properties of $\alpha$ - and $\beta$-chains from human hemoglobin. J. Biol. Chem. 244: 4664.

11. Clegg, J. B., M. A. Naughton, and D. J. Weatherall. 1966. Abnormal human haemoglobins. Separation and characterization of the $\alpha$ and $\beta$ chains by chromatography, and the determination of two new variants, $\mathrm{Hb}$ Chesapeake and Hb J (Bangkok). J. Mol. Biol. 19: 91.

12. Ambler, R. P. 1967. Enzymic hydrolysis with carboxypeptidases. Methods Enzymol. 11: 155.

13. Duvelleroy, M. A., R. G. Buckles, S. Rosenkaimer, C. Tung, and M. B. Laver. 1970. An oxyhemoglobin dissociation analyzer. J. Appl. Physiol. 28: 227.
14. Bunn, H. F., and R. W. Briehl. 1970. The interaction of 2,3-diphosphoglycerate with various human hemoglobins. J. Clin. Invest. 49: 1088.

15. Wintrobe, M. M. 1967. Clinical Hematology. Lea \& Febiger, Philadelphia. 6th edition. 86.

16. Dacie, J. V., A. J. Grimes, A. Meisler, L. Steingold, E. H. Hemsted, G. H. Beaven, and J. C. White 1964. Hereditary Heinz-body anaemia. A report of studies on five patients with mild anaemia. Br. J. Haematol. 10: 388.

17. Benesch, R. E., R. Benesch, and C. I. Yu. 1969. The oxygenation of hemoglobin in the presence of 2,3-diphosphoglycerate. Effect of temperature, $\mathrm{pH}$, ionic strength and hemoglobin concentration. Biochemistry. 8: 2567.

18. Sevringhaus, J. W. 1966. Blood gas calculator. J. Appl. Physiol. 21: 1108.

19. Bradley, T. B., Jr., R. C. Wohl, and R. F. Rieder. 1967. Hemoglobin Gun Hill: deletion of five amino acid residues and impaired heme-globin binding. Science (Wash. D. C.). 157: 1581 .

20. Wyman, J., Jr. 1964. Linked functions and reciprocal effects in hemoglobin: a second look. Adv. Protein Chem. 19: 223.

21. Novy, M. J., M. J. Edwards, and J. Metcalfe. 1967. Hemoglobin Yakima. II. High blood oxygen affinity associated with compensatory erythrocytosis and normal hemodynamics. J. Clin. Invest. 46: 1848.

22. Adamson, J. W., J. T. Parer, and G. Stamatoyannopoulos. 1969. Erythrocytosis associated with hemoglobin Rainier: oxygen equilibria and marrow regulation. $J$. Clin. Invest. 48 : 1376.

23. Lehmann, H., and R. W. Carrell. 1968. Differences between $\alpha$ - and $\beta$-chain mutants of human haemoglobin and between $\alpha$ - and $\beta$-thalassaemia. Possible duplication of the $\alpha$-chain gene. Br. Med.J. $4: 748$.

24. Carrell, R. W., and H. Lehmann. 1969. The unstable haemoglobin haemolytic anaemias. Semin. Hematol. 6: 116.

25. Fitch, W. M. 1967. Evidence suggesting a non-random character to nucleotide replacements in naturally occurring mutations. J. Mol. Biol. 26: 499.

26. Vogel, F. 1969. Point mutations and human hemoglobin variants. Humangenetik. $8: 1$.

27. Ohta, T., and M. Kimura. 1971. Amino acid composition of proteins as a product of molecular evolution. Science (Wash. D. C.). 174: 150.

28. Tyuma, I., K. Imai, and K. Shimizu. 1971. Effect of inositol hexaphosphate and other organic phosphates on the cooperativity in oxygen binding of human hemoglobins. Biochem. Biophys. Res. Commun. 44: 682.

29. Morimoto, H., H. Lehmann, and M. F. Perutz. 1971. Molecular pathology of human haemoglobin: stereochemical interpretation of abnormal oxygen affinities. Nature (Lond.). 232: 408.

30. Edelstein, S. J. 1971. Extensions of the allosteric model for haemoglobin. Nature (Lond.). 230: 224.

31. Charache, S., D. J. Weatherall, and J. B. Clegg. 1966. Polycythemia associated with a hemoglobinopathy. $J$. Clin. Invest. $45: 813$.

32. Nagel, R. L., Q. H. Gibson, and S. Charache. 1967. Relation between structure and function in hemoglobin Chesapeake. Biochemistry. $6: 2395$.

33. Bunn, H. F. 1970. Dissociation of haemoglobin Chesapeake into subunits. Nature (Lond.). 227: 839. 
34. Bunn, H. F., B. J. Ransil, and A. Chao. 1971. The interaction between erythrocyte organic phosphate, magnesium ion, and hemoglobin. J. Biol. Chem. 246: 5273.

35. Dacie, J. V., N. K. Shinton, P. J. Gaffney, Jr., R. W. Carrell, and H. Lehmann. 1967. Haemoglobin Hammersmith ( $\beta 42$ (CD1) Phe $\rightarrow$ Ser). Nature (Lond.). 216: 663.

36. Miller, D. R., R. I. Weed, G. Stamatoyannopoulos, and A. Yoshida. 1971. Hemoglobin Köln disease occurring as a fresh mutation: erythrocyte metabolism and survival. Blood J. Hematol. 38: 715.

37. Fairbanks, V. F., R. W. Opfell, and E. O. Burgert, Jr. 1969. Three families with unstable hemoglobinopathies (Köln, Olmstead and Santa Ana) causing hemolytic anemia with inclusion bodies and pigmenturia. Am. J. Med. $46: 344$.

38. Hollán, S. R., J. G. Szelélenyi, M. Miltenyi, D. Charlesworth, R. A. Lorkin, and H. Lehmann. 1970. Unstable haemoglobin disease caused by Hb Santa Ana $\beta 88$ (F4) Leu $\rightarrow$ Pro. Haematologia. 4: 141.

39. Jones, R. T., B. Brimhall, T. H. J. Huisman, E. Kleihauer, and K. Betke. 1966. Hemoglobin Freiburg: abnormal hemoglobin due to deletion of a single amino acid residue. Science (Wash. D. C.). 154: 1024.

40. Schneider, R. G., S. Ueda, J. B. Alperin, B. Brimhall, and R. T. Jones. 1969. Hemoglobin Sabine $\beta 91$ (F7) Leu $\rightarrow$ Pro. An unstable variant causing severe anemia with inclusion bodies. N. Engl. J. Med. 280: 739.

41. Huisman, T. H. J., A. K. Brown, G. D. Efremov, J. B.
Wilson, C. A. Reynolds, R. Uy, and L. L. Smith. 1971. Hemoglobin Savannah ( $\beta 6$ (24) $\beta$-glycine $\rightarrow$ valine): an unstable variant causing anemia with inclusion bodies. J. Clin. Invest. 50: 650 .

42. Cabannes, R., D. Labie, J. Rosa, J. Ruffié, and R. Bierme. 1969. Une "nouvelle" hémoglobine par mutation portée sur la chaine $\beta$, l'hémoglobine I Toulouse $\left(\alpha_{2}-\right.$ $\left.\boldsymbol{\beta}_{2}{ }^{6{ }^{158} \rightarrow g^{14}}\right)$. C. R. Hebd. Seances Acad. Sci. Ser. D. Sci. Nat. (Paris). 269: 111.

43. Steadman, J. H., A. Yates, and E. R. Huehns. 1970. Idiopathic Heinz body anaemia: Hb Bristol ( $\beta 67$ (E11) Val $\rightarrow$ Asp). Br. J. Haematol. 18: 435.

44. Josephson, A. M., H. G. Weinstein, V. J. Yakulis, L. Singer, and P. Heller. 1962. A new variant of hemoglobin $\mathbf{M}$ disease: hemoglobin M-Chicago. J. Lab. Clin. Med. 59 : 918.

45. Farmer, M. B., H. Lehmann, and D. N. Raine. 1964 Two unrelated patients with congenital cyanosis due to haemoglobinopathy M. Lancet. 2 : 786.

46. Koler, R. D., R. T. Jones, M. Duerst, and Z. Stocklen.

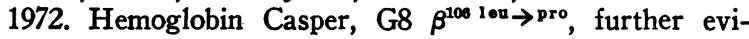
dence that hemoglobin mutations are not random. In Second International Conference on Red Cell Metabolism and Function. G. Brewer, editor. Plenum Publishing Corporation, New York. In press.

47. Becroft, D. M. O., R. Douglas, R. W. Carrell, and H. Lehmann. 1968. Haemoglobin M-Hyde Park: a hereditary methaemoglobinaemia in a Caucasian child. $N . Z$. Med.J. 68: 72 . 